Index: chemistry, scientific instruments, soil sciences, solid-state physics

\title{
Thermoluminescence: Potential Applications in Forensic Science
}

\author{
J. D. Ingham and D. D. Lawson
}

Propulsion Division

\begin{abstract}
In crime laboratories one of the most difficult operations is to determine unequivocally whether or not two samples of evidence of the same type were originally part of the same thing or were from the same source. It has been found that high temperature thermoluminescence (room temperature to $723 \mathrm{~K}$ ) can be used for comparisons of this type, although work to date indicates that there is generally a finite probability for coincidental matching of glass or soil samples. Further work is required to determine and attempt to minimize these probabilities for different types of materials, and to define more clearly the scope of applicalility of thermoluminescence to actual forensic situations.
\end{abstract}

\section{Introduction}

The criminalistics operation in the investigative process is to identify, evaluate, and interpret potential evidence or physical materials associated with crime situations. These materials frequently offer the most convincing means of relating a suspect to an act or a scene, or of showing that a person has been incorrectly suspected.

Methods that may be available to criminalists and that have been applied to evidence materials include conventional chemical analysis, optical microscopy, gas chromatography, spectrometry, and measurements of physical properties such as refractive index and density. When applying these techniques the criminalist usually compares the physical materials found at the scene of a crime, or known to be related to a crime, with those known to be related to the suspect. He then attempts to demonstrate convincing relationships, either negative or positive, from the results.

Because of practical limitations it is very often not possible to say unequivocally that two materials were derived from the same source, since no two things are exactly alike. In such cases the criminalist must examine all information available and then make an accurate estimate of the situation based on his past experience. Except for such evidence as clear fingerprints, 
very characteristic bullet rifling patterns, or other special cases, most evidence materials fall in the category described above. That is, the criminalist requires all the information that he can obtain to effectively compare and evaluate evidence materials.

Therefore, there is a critical need for new methods and instruments that can be used for the accurate interpretation of physical evidence, not to replace those techniques already in use, but to extend and enlarge the capabilities of forensic practitioners to individualize or convincingly assess the source relationships of evidence materials.

From the work carried out at JPL, it appears that thermoluminescence (TL) is one of the more discriminating methods available for differentiating between specific samples of several type of materials, including glass, soils, safe insulation, salts, and other nonmetallic solids. It was recently pointed out that a national survey of criminalists has indicated that improved methods are needed most for glass, hair, paint, soil, fibers, and blood, in that order (Ref. 1); therefore, it is believed that the application of TL methods would be of significant value for the investigation of a number of important types of evidence materials.

\section{Thermoluminescence: Technical}

Thermoluminescence (TL) is the emission of light that may occur when a material is heated to temperatures below incandescence (Ref. 2). The primary cause of these light emissions is previous exposure of the material to natural or laboratory ionizing radiation at or below the temperature at which heating is started. The radiant energy displaces some electrons in the solid; these electrons are trapped in imperfections and vacancies in the crystal lattice and escape with the emission of light when the temperature is raised to supply the required amount of kinetic energy. The color or wave length distribution of the light may vary over the entire visible spectrum and appears at different temperatures depending on the basic crystal structure, the presence of impurities or trace components, and the radiation, thermal, and pressure histories of the material.

For high temperature TL, which is the subject of this work, the temperature range is from room temperature to $723 \mathrm{~K}\left(450^{\circ} \mathrm{C}\right)$. TL response is recorded in about two minutes as temperature vs light intensity to give a TL curve. Since the responses, even for relatively pure materials, depend on several subtle variable factors such as concentrations of minor components and environmental history, the shapes of the TL curves often depend on the source of the particular material. For applications to criminalistics, TL response for a specific material is compared with the curve obtained under the same conditions for another sample of the same substance of known origin. If the curves are essentially identical, a common source is indicated for the two materials being compared. 


\section{Thermoluminescence: Literature}

TL was probably first described in the last half of the seventeenth century by Robert Boyle. In 1928 the coloration of glass by radioactivity followed by heating to bleach the color and evolve light was described by Lind (Ref. 3). Also in the twenties, the TL responses of fluorite and carbonate minerals were observed (Refs. 4 and 5). It was noted that after once being heated these materials would not again emit light on heating, but that TL could be regenerated by exposure to $\mathrm{X}$-rays or radium. Urbach (Ref. 6) and Randall and Wilkins (Ref. 7) recorded glow curves and interpreted them theoretically.

Between 1948 and 1959, F. Daniels and others carried out a large amount of work on potential applications of TL (Ref. 8). These included applications to uranium prospecting, radiation damage and dosimetry, geological age determinations of minerals and rocks, stratigraphy, catalysis, and identification and control of ceramic materials. Good results have been obtained by TL for archeological dating of pottery (Refs. 9 and 10). Since the intensity of TL of a sample depends on the extent of high temperature drainage, it has been used as a paleoclimatological tool to determine the time for glaciation of antarctic regions and to estimate microclimatic effects (Refs. 11 and 12). Although most TL investigations have been concerned with fairly welldefined minerals and materials, Nishita and Hamilton have studied a number of soil samples, especially from the point of view of using soils as radiation dosimeters (Ref. 13), and a limited study of the stratigraphy of lunar samples by TL has been carried out (Ref. 14). At JPL over 100 antarctic dry valley soil samples have been investigated by TL mainly to attempt to correlate microclimate and soil properties with TL responses, which were then related to microbial abundances. (Refs. 15 and 16).

Although there appear to be no previous investigations directed toward applications of TL to criminalistics, theoretical considerations and much of the work described in the literature indicate that TL responses are characteristic and may depend on the source of a particular material. For example, four samples of potassium bromide from different sources gave decidedly different glow curves (Ref. 17). Differences characteristic of the source have also been observed for glass, clay, soils, seashore sand, and calcite (Refs. 2, 18 and 19).

\section{Results and Discussion}

The scope of applications of TL to forensic investigations has not yet been fully defined. Therefore, this is essentially a progress report, with emphasis on the status of current methodology for high temperature TL (room temperature to $723 \mathrm{~K}\left(450^{\circ} \mathrm{C}\right)$ ) as applied to materials such as glass and soil samples.

The samples were prepared by mild crushing or grinding, sieved to reasonably uniform particle size and exposed to laboratory irradiation. All samples to be compared were exposed to the same dose of ionizing radiation 
from a Cs 137 or Co 60 source. The TL curves were then recorded by heating each sample $(\sim 7 \mathrm{mg})$ at a rapid rate $(\sim 9 \mathrm{~K} / \mathrm{s})$ and measuring the light evolved vs temperature using a TL photometer (Fig. 1). The light intensity range covered four decades from about 0.01 to $10 \mu / \mathrm{m}$, but was expressed in arbitrary units. A view of the current sample cup and resistance heated support ribbon is shown in Fig. 2. The samples were volumetrically loaded into the cup by means of a microspoon. If the curves were exactly the same, or very different, they could be compared visually (cf Figs. 3 and 4). However, for curves that were questionably similar, or for comparisons of all combinations of more than two or three curves, a statistical method was used (Ref. 20).

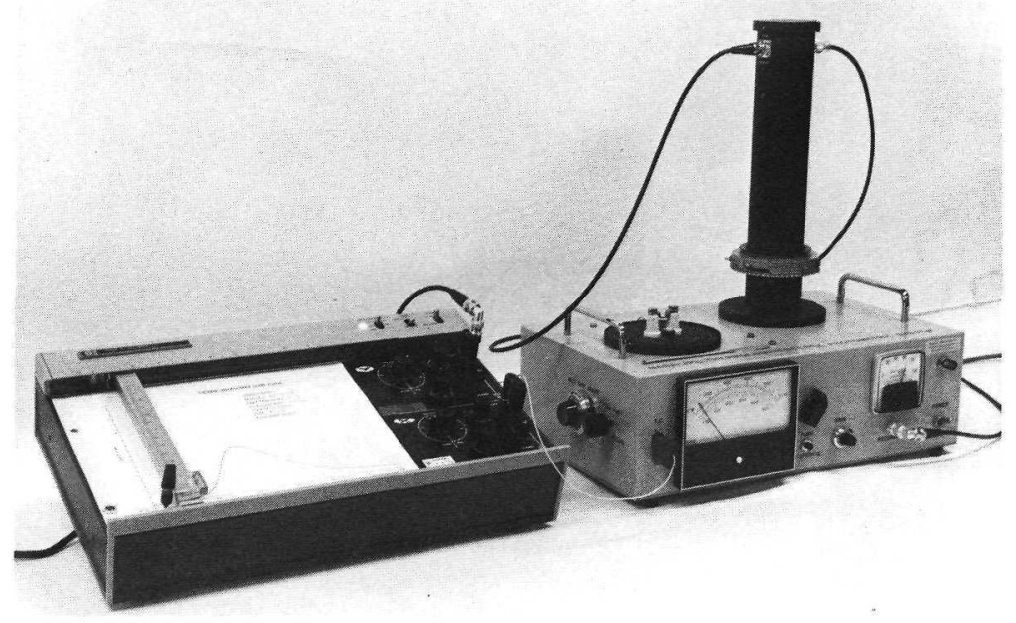

Fig. 1. Thermoluminescence photometer

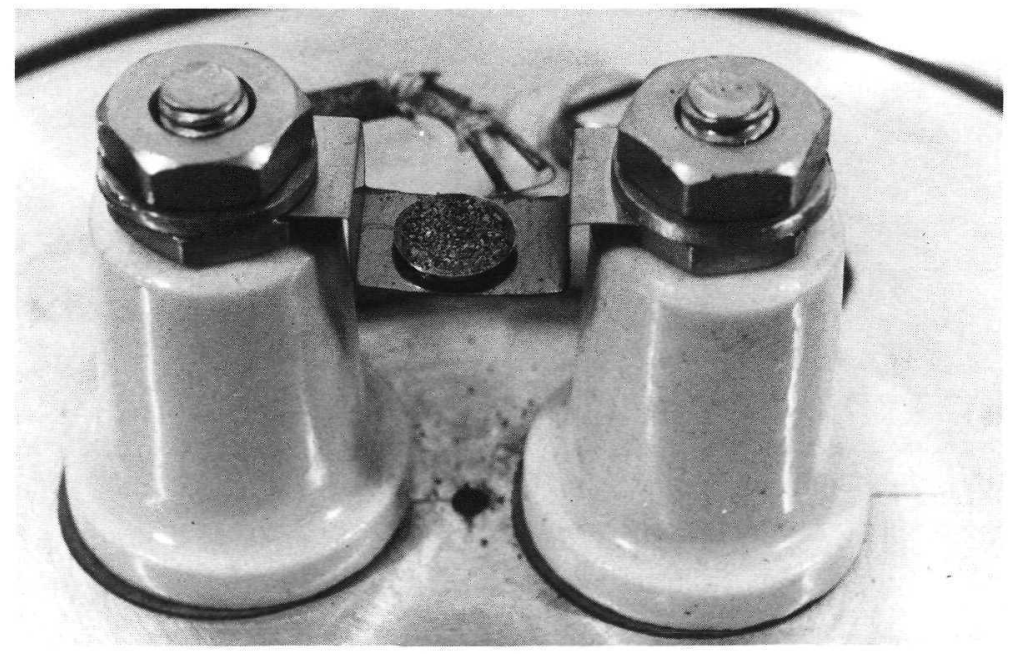

Fig. 2. Thermoluminescence sample cup and heater 


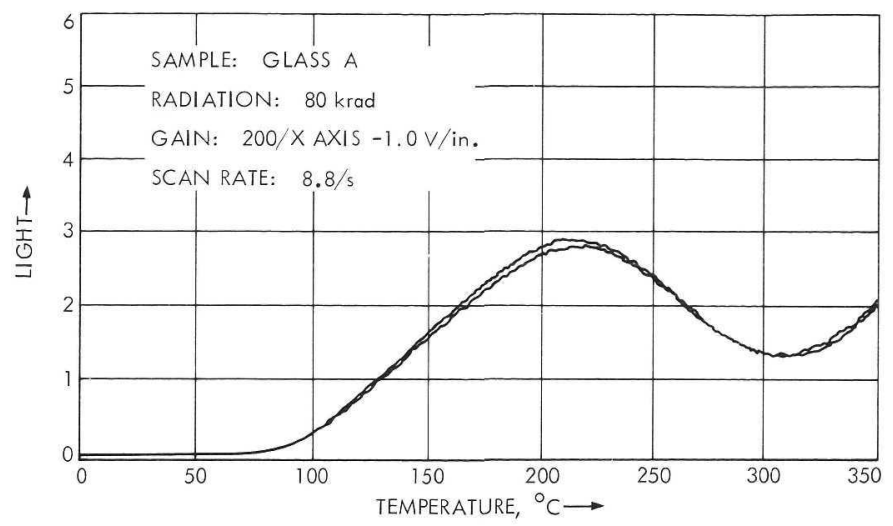

Fig. 3. Thermoluminescence curve for glass sample (A)

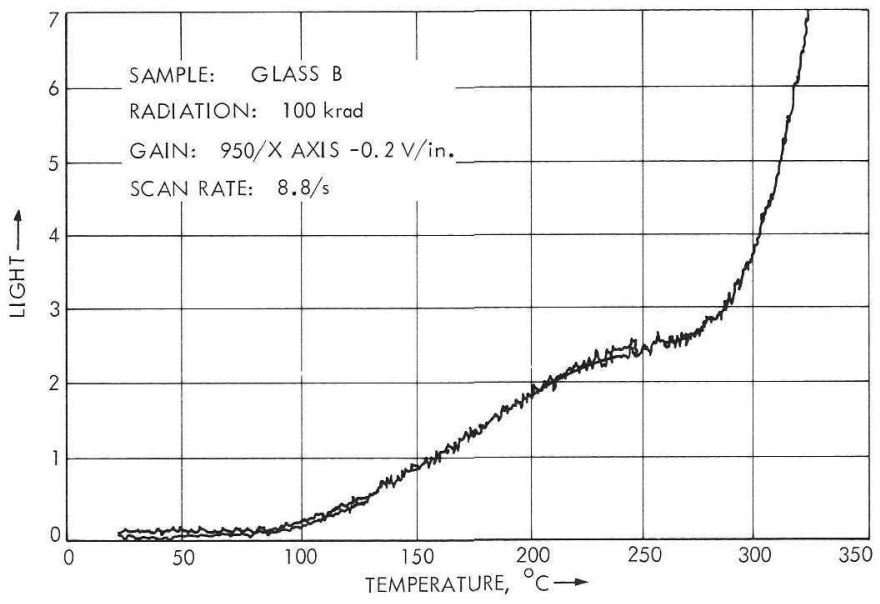

Fig. 4. Thermoluminescence curve for glass sample (B)

For an initial estimate of the utility of TL in forensic situations, data for ten auto headlamp lenses were obtained and compared by the $\chi^{2}$ method (Ref. 20). The threshold value of $\chi^{2}$ was determined from only one headlamp, designated AC No. 4201.

The values of $\chi^{2}$ are used to compare curves; each $\chi^{2}$ value represents the magnitude of the absolute differences in areas between the two TL curves being compared. These values are calculated from:

$$
\chi^{2}=\sum \frac{(E-O)^{2}}{E}
$$


in which $\mathrm{E}$ and $\mathrm{O}$ are the expected and observed events, respectively. The two curves to be compared are divided into 33 equal parts at $10 \mathrm{~K}$ intervals. The differences in light intensities at each interval are then measured from the chart to give 33 values of $\mathrm{E}$ - O. Since there is no rationale for defining which curve represents expected events or which represents observed events, $\mathrm{E}$ and $\mathrm{O}$ are interchanged in Eq. (1) to calculate two $\chi^{2}$ values for each comparison of two curves. It is obvious that the larger the $\chi^{2}$ values, the greater the difference between the two curves, and, if the curves are the same, $\chi^{2}$ equals 0 . Comparison of curves obtained from several measurements on samples from the same source results in $\chi^{2}$ values that are low, and reflect the reproducibility of the TL method.

A series of $\chi^{2}$ values calculated from TL measurements on four different glass samples taken from different parts of the same AC No. 4201 lens are shown in the matrix below.

0.101

0.096

0.429

0.597

0.472

0.421

0.494

0.151

0.366

0.165

Samples (1), (2), (3), and (4) were taken from the central, right, left, and lower parts of the lens, respectively. For comparisons among four curves, $\chi^{2}$ values have been calculated from all possible combinations of the four, to give six pairs of values (that is; $n(n-1) / 2$ equals 6.) By using probability paper, the mean $(\mathrm{X})$ and standard deviation $(\mathrm{S})$ were found to be 0.42 and 0.28 , respectively. These could have been directly calculated with slightly more accuracy, but the probability plot was convenient and showed that the data could be reasonably well represented as a normal distribution. The threshold value of $\chi^{2}$ was used as a criterion for curve matching. Thus, if $\chi^{2}$ for a comparison of two curves for different lens glasses was less than the threshold value of $\chi^{2}$, a match of the two curves was indicated. In the example above, the threshold value was $\chi^{2}$ equals 1.26 , obtained by adding three times the standard deviation to the mean. Three $S$ was added to the mean because it has been found that for a normal distribution about $99.7 \%$ of the measurements fall in the range of $\mathrm{X} \pm 3 S$; thus, using only the four TL curves nearly all TL curves for lens AC No. 4201 should have $\chi^{2}$ values of less than 1.26 when compared with a lens sample known to be from this lens. However, it has been shown by Ojena, et al. (Ref. 21), that variations in refractive index for the same auto headlamp lenses occur, probably because of differences in the annealing schedule at different positions of the lens. In agreement with their work, it was found that the $\chi^{2}$ value for a sample taken from the edge of lens AC No. 4201 was greater than 1.26 in two comparisons with the four AC No. 4201 samples discussed above. If this TL curve was 
included in the calculation of the threshold $\chi^{2}$, the value was found to be 2.41. Since there is no reason to exclude this last sample, the larger threshold value was used for the comparisons of headlamp glasses shown in Table 1. It can be seen that sample 1 matches 2 and 6; 2 matches 6 ; and 3 matches 5 and 6 , which represents $89 \%$ success in differentiating among auto headlamp samples.

Table 1. Matrix of $\chi^{2}$ values for different headlight lens samples

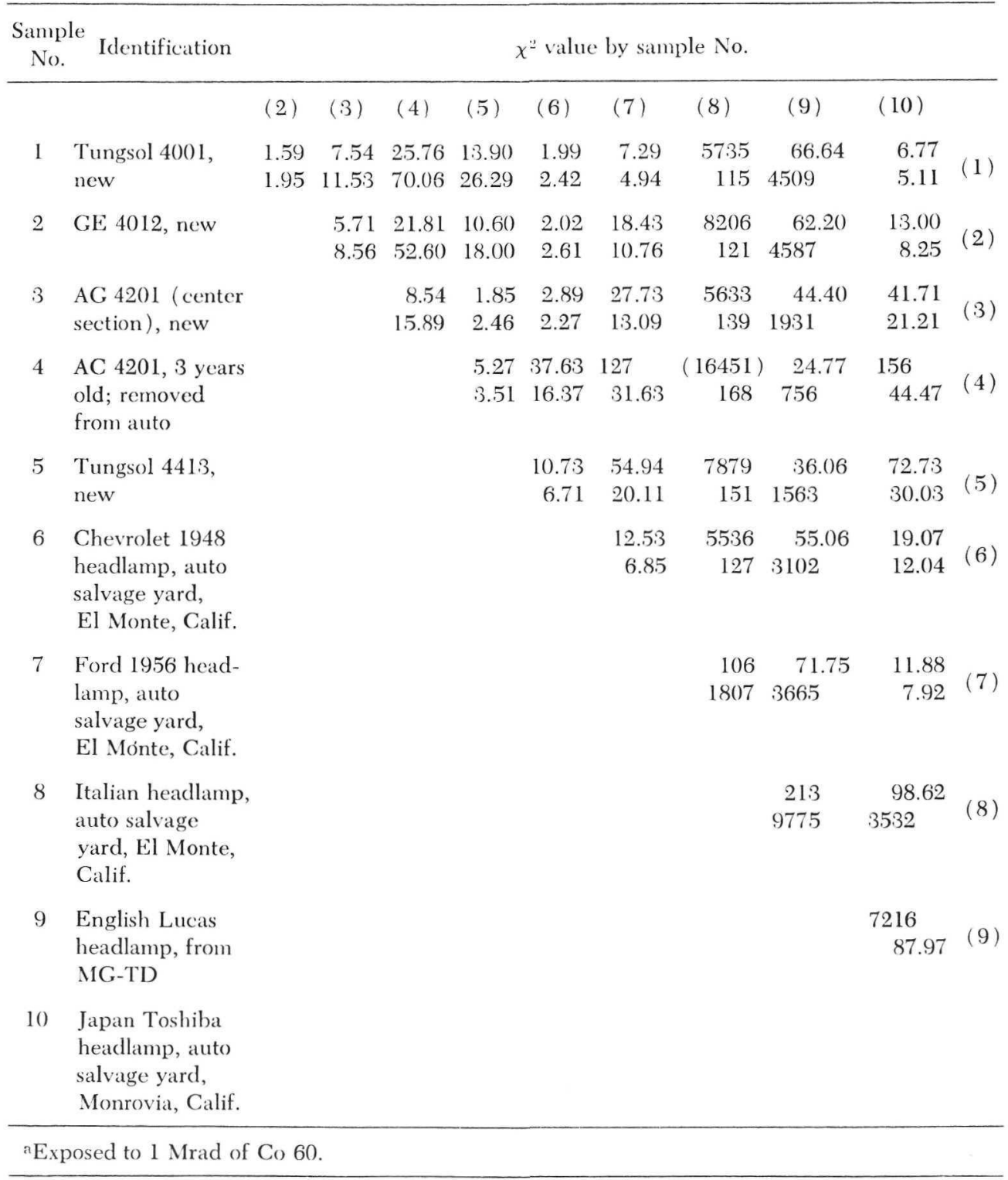


The above results clearly show that TL response is not a unique characteristic of auto headlamp samples at the present stage of development, and that the probability of coincidental matching can be quite high. If a threshold of $\mathrm{X}+3 \mathrm{~S}$ is used it appears that TL can only be used for exclusionary evidence, i.e., where the TL curves obviously do not match, or for preliminary investigations that might lead to further substantial evidence.

However, in the context of actual cases, it may be practical to decrease the threshold value. For example, when a threshold value of $\mathrm{X}+\mathrm{S}$ equals 1.31 was used for the headlamp lens data in Table 1 , no coincidental matches were observed in the 45 comparisons. But this procedure would result in the observation of about $16 \%$ negative comparison results for samples obtained from the same material. Thus, by using a lower threshold value, existing data indicate that coincidental positives can be greatly decreased, but that about one of six true positives would incorrectly appear to be negative. At least in those cases where positive matching would be incriminating, the assignment of a lower threshold value may be justified, since the loss of some evidence is not as serious as the application of invalid evidence.

In six comparisons of windshield glasses, the $\chi^{2}$ values for one comparison were $0.69,1.72$, which is a true coincidental match even if the threshold was substantially less than 2.4. Similarly, for three comparisons of auto door glass there was one coincidental match $\left(\chi^{2}\right.$ equals $\left.0.75,1.12\right)$. Also for 21 comparisons of vodka bottles (all of the same vodka brand), there were $5 \chi^{2}$ values less than 1.3 , or $24 \%$ of the comparisons were coincidental matches. In this example, there were no $\chi^{2}$ values between 1.3 and 2.4 ; therefore, either threshold would give the same results.

A threshold value of 1.8 was determined for building window glass and the $\chi^{2}$ values were calculated for two samples. All three comparisons resulted in $\chi^{2} \mathrm{~s}$ that were well above the threshold values. Four additional samples all required different levels of irradiation to induce thermoluminescence, indicating there were no matches among these other four samples. These results show that TL may be more satisfactory for applications to building glass than for auto glass, but a larger statistical sample will be required for confirmation.

The situation is much more favorable for soil samples. Out of 630 comparisons involving 36 samples, there were only $15(2.38 \%)$ coincidental matches, and these were obtained using a predetermined relatively high threshold $\chi^{2}$ value of 3.30 . Furthermore, after making the comparisons it was found that only 6 of the 15 matching comparisons were purely coincidental: two of the remaining samples were taken from the same location, and the other matching pairs were taken from slightly different depths at the same location; thus the true coincidental matches involved only $\sim 1 \%$ of the total number of comparisons. There were only four totally coincidental positive comparisons if the threshold $\chi^{2}$ value was taken as $\mathrm{X}+$ $S=2.52$. Since all of these samples were collected from one northern California county, these results should not be applied universally without 
further confirmation. For example, it is possible that surface samples taken from a relatively large, rolling dune or desert area may give TL curves that are much more alike than the above results would predict.

\section{Conclusions}

Thermoluminescence appears to be a useful technique for the interpretation and evaluation of certain types of physical evidence materials. The degree of discrimination among soils is greater than for glass samples, which probably arises because of the standardization and quality control applied in the manufacture of glass, and its more constant composition. Preliminary work indicates that for most other materials, such as salts, relatively pure minerals and safe insulation, the overall differences in TL curves for each type of material are intermediate between glass and soils.

There are a number of things that should be done to further define the scope of applications and improve the methodology of TL. For example, the amount of data should be greatly increased to provide greater reliability in the statistical evaluation of results for different types of samples, preferably by criminalists who would be more familiar with potential forensic situations. There are also some instrumental and procedural modifications that may be warranted. These include examination of the effects of prior sample annealing, simple modifications of the sample pan to facilitate uniform loading, and inclusion of measurements of spectral distribution of TL light to greatly decrease the probability for coincidental matching of unrelated samples. These spectral measurements are feasible, either by obtaining a series of TL curves for each sample with different filters between the sample pan and detector, or by using a more elaborate TL photometer that records a series of spectra at small temperature intervals between room temperature and $723 \mathrm{~K}$.

\section{References}

1. Kingston, C. R., Report to the Criminalistics Subcommittee of ASTM Committee E-30 on Forensic Science, Phoenix, Arizona, Feb. 22, 1971.

2. Daniels, F., Boyd, C. A., and Saunders, D. F., "Thermoluminescence as a Research Tool", Science, Vol. 117, pp. 343-349, 1953.

3. Lind, S. C., The Chemical Effects of Alpha Particles and Electrons, p. 29, Reinhold's, New York, 1928.

4. Wick, F. G., Phys. Rev., Vol. 25, p. 588, 1925.

5. Wick, F. G., J. Opt. Soc. Am., Vol. 21, p. 223, 1931.

6. Urbach, F., Aksd. Wiss. Wein. Ber., Vol. 139, No. 20, p. 354, 1930.

7. Randall, J. T., and Wilkins, M. E. F., Proc. R. Soc., Vol. A184, p. 366, 1945 . 
8. Daniels, F., "Early Studies of Thermoluminescence in Geology", in Thermoluminescence of Geological Materials. Edited by D. J. McDougall, pp. 3-12, Academic Press, New York, 1968.

9. Mazess, R. B., and Zimmerman, D. W., "Thermoluminescence Dating of Some Peruvian Pottery", in Thermoluminescence of Geologic Materials. Edited by D.J. McDougall, pp. 445-448, Academic Press, New York, 1968.

10. Ralph, E. K., and Han, M. C., "Dating of Pottery by Thermoluminescence”, Nature, Vol. 210, pp. 245-247, 1966.

11. Ronca, L. B., "Thermoluminescence as a Paleo-Climatological Tool”, in Thermoluminescence of Geological Materials. Edited by D. J. McDougall, pp. 495-506, Academic Press, New York, 1968.

12. Zeller, A. N., “The Influence of Microclimate Upon the Thermoluminescence of Rock", in Thermoluminescence of Geologic Materials. Edited by D.J. McDougall, pp. 507-518, Academic Press, New York, 1968.

13. Nishita, H., and Hamilton, M., "Spurious Thermoluminescence of Soils”, Soil Science, Vol. 110, pp. 371-378, 1970.

14. Doell, R. R., and Dalrymple, G. B., "Thermoluminescence of Apollo 12 Lunar Samples", Earth and Planetary Science Letters, Vol. 10, pp. 357-360 1971.

15. Cameron, R. E., and Benoit, R. E., "Microbial and Ecological Investigations in Victoria Dry Valley, Southern Victoria Land, Antarctica”, Antarctic Research Series, American Geophysical Union, Antarctic Terrestial Biology, in press.

16. Ingham, J. D., Cameron, R. E., and Lawson, D. D., "Microbial Abundance and Thermoluminescence of Antarctic Dry Valley Soils", Soil Science, in press.

17. Fieschi, R., and Scaramelli, P., "Photostimulated Thermoluminescence in Alkali Halide Crystals", in Thermoluminescence of Geological Materials. Edited by D. J. McDougall, pp. 291-308, Academic Press, New York, 1968.

18. Mejdahl, V., "Thermoluminescence in Seashore Sand", in Thermoluminescence of Geological Materials. Edited by D. J. McDougall, pp. 453-462, Academic Press, New York, 1968.

19. Medlin, W. L., "Thermoluminescence Growth Curves in Calcite", in Thermoluminescence of Geologic Materials. Edited by D. J. McDougall, pp. 91-101, Academic Press, New York, 1968.

20. Lawson, D. D., and Framan, E. P., "Numerical Correlation and Evaluation in the Comparison of Evidentiary Materials," J. Forensic Sci., in press. 
21. Ojena, S. M., DeForest, P. R., and Crim, D., “A Study of Refractive Index Variations Within and Between Sealed Beam Headlights Using a Precise Method", J. Forensic Sci., Vol. 17, No. 3, pp. 409-425, 1972. 